Research Article

\title{
Oxytocin injection quality in Ethiopia: a post-marketing surveillance study in public and private facilities across three regions
}

\author{
Pete Lambert ${ }^{1}$, Tri-H Nguyen', Victoria L Oliver ${ }^{1}$, Andrew J L McArthur ${ }^{1}$, Cleo Goodall', Alula M Teklu², Bikila Bayissa ${ }^{3}$ \\ Zelalem Mamo ${ }^{4}$, Delayehu Bekele ${ }^{5}$, Michelle P McIntosh \\ 1 Drug Delivery, Disposition and Dynamics, Monash Institute of Pharmaceutical Sciences, Monash University, Parkville, Victoria, Australia, ${ }^{2}$ MERQ \\ Consultancy, Addis Ababa, Ethiopia, ${ }^{3}$ Food, Medicines and Health Care Administration and Control Authority of Ethiopia, Addis Ababa, Ethiopia, 4 \\ United States Pharmacopeial Convention - Promoting Quality of Medicines Programme, Addis Ababa, Ethiopia, ${ }^{5}$ Department of Obstetrics and \\ Gynaecology, St Paul's Hospital Millennium Medical College, Addis Ababa, Ethiopia \\ Keywords: global health \\ https://doi.org/10.29392/joghr.3.e2019081
}

\section{Journal of Global Health Reports}

Vol. 3, 2019

\section{Background}

The high prevalence of poor quality essential medicines in low and middle income countries (LMIC) presents considerable risks in terms of both health outcomes and economic cost. Oxytocin injection, the gold standard therapy for management of postpartum haemorrhage $(\mathrm{PPH})$, presents a particular challenge in this area. Recent studies in India, Nigeria and DRC have identified product failure rates, in terms of low drug content, to be $41 \%, 74 \%$ and $80 \%$ respectively. Ethiopia bears a high burden of PPH with over $40 \%$ of maternal deaths being directly attributed to haemorrhagic causes. This study assessed the quality of oxytocin injection at points in the public and private supply chains to support national efforts to address PPH in Ethiopia.

\section{Methods}

This study sampled oxytocin injection ampoules from 45 sites across Oromia, Afar regions and the administrative area of Addis Ababa. This included points along the public supply chain from the national point of entry for supplies through regional hubs to points of use (public and private facilities) in urban and rural areas. Collected samples were stored under refrigerated conditions until analysis for oxytocin content, known degradation products and microbiological quality.

\section{Results}

Ninety-six percent of ampoules passed all tests, while two samples (4\%) contained less than the specified oxytocin content. Both samples were collected from rural facilities in Afar, a remote, poorly resourced region with a very hot climate. All supplies collected were sourced from European stringent regulatory approved (SRA) suppliers and, where storage conditions could be determined, approximately $95 \%$ of samples were stored in the refrigerator at the time of collection.

\section{Conclusions}

The study indicates that oxytocin injection in the selected regions is generally of high quality and being stored appropriately. The failed samples detected in Afar suggest challenges remain around maintenance of refrigerated storage in least resourced settings. These findings contrast with recent results in other African countries and support the joint World Health Organization (WHO)/United Nations Children Fund/United Nations Population Fund statement that the availability of high quality oxytocin injection at the point of use is dependent on procurement of supplies from manufacturers approved by WHO or SRAs and storage at $2-8^{\circ} \mathrm{C}$ throughout the supply chain.

Recent studies have highlighted the issue of substandard and falsified (SF) medicines in low and middle income countries (LMICs). A systematic review estimated the prevalence of SF medicines in these regions to be $13.6 \%$ with higher rates in some areas (Africa 18.7\%) and therapeutic categories (antimalarials 19.1\%). ${ }^{1}$ Quantifying the potential economic burden caused by SF medicines is challenging as detailed information is limited, however the same study es- 
timated the annual financial impact to be between US\$ 10 billion to US\$200 billion.

Oxytocin injection, the first line therapy for both the prevention and treatment of postpartum haemorrhage (PPH), presents particular challenges in LMICs in terms of the maintenance of high quality products throughout the supply chain. ${ }^{2}$ These challenges result in part from the requirement for all oxytocin injection to be stored under refrigerated conditions (ie, $2^{\circ} \mathrm{C}-8^{\circ} \mathrm{C}$ ) for the duration of the product shelf-life. ${ }^{3}$ In low resource settings, the infrastructure to maintain consistent cold chain supply and storage is often lacking, potentially leading to product deterioration.

In addition, there are a high number of oxytocin injection manufacturers that supply ampoules into the global market (reportedly in excess of 100, with possibly as many as 300 products). ${ }^{4}$ Many of these suppliers operate in countries that do not adhere to stringent manufacturing quality standards and will supply into countries where marketing authorisations have not been obtained. ${ }^{5}$ Consequently, the quality of oxytocin ampoules at the time of manufacture varies considerably between suppliers, with inappropriate labelling around storage conditions and high impurity levels being examples of identified deficiencies. ${ }^{6}$

As a result of these factors, multiple studies have found that the quality of oxytocin products available in low and middle income countries does not consistently meet internationally recognised standards. Torloni et al. conducted a systematic review of studies examining the quality of oxytocin in Africa, Asia and Latin America. ${ }^{7}$ Four eligible studies conducted evaluations in eight African countries and found a mean of $57.5 \%$ of ampoules did not contain the specified content of oxytocin. Subsequent studies in Nigeria and the Democratic Republic of Congo showed a higher incidence of ampoules failing to meet content specifications. 6,8

Given these findings and that $\mathrm{PPH}$ is estimated as the leading global cause of pregnancy-related deaths (approximately 20\%), with these deaths occurring overwhelmingly in the low and lower-middle income countries, international efforts are focussing on strategies to improve oxytocin quality. 3,9

Ethiopia is a country that bears a high burden of maternal mortality. World Health Organisation data estimates that 11,000 maternal deaths occurred in Ethiopia in 2015, making it the fourth largest contributor to the total number of maternal deaths worldwide. ${ }^{10}$ A recent Federal Ministry of Health (FMoH) Maternal Death Surveillance and Response initiative (Ethiopian Public Health Institute, 2016) highlighted that just over $30 \%$ of maternal deaths in the country are due to $\mathrm{PPH} .{ }^{11}$ With an aim to improve outcomes, the Government of Ethiopia has targeted maternal health as a major priority in its Health Sector Transformation Plan (2015/2016 - 2019/2020), and specific focus has been directed toward addressing the burden of PPH. ${ }^{12}$

Although the national medicine regulatory authority has undertaken small scale oxytocin post-marketing surveillance sample testing in previous years, no published studies have examined the quality of oxytocin ampoules in Ethiopia. Oxytocin injection quality may be compromised at the point of entry into the country (due to substandard manufacture) and/or at the point of use, particularly in low resource regions where distances to rural facilities are great and supporting infrastructure to maintain controlled supply and storage can be poor.

This study, conducted in partnership with Ethiopian government authorities, aimed to assess the quality of oxytocin ampoules at a number of healthcare facilities and distribution/supply outlets in selected areas of Ethiopia. The study used purposeful sampling in two regions (Oromia and Afar) and one administrative centre (Addis Ababa) to i) better understand the oxytocin injection quality landscape and ii) guide government initiatives to address $\mathrm{PPH}$, inform future research and support global efforts to address poor oxytocin quality.

\section{METHODS}

Methodology and results have been reported according to World Health Organization (WHO) Guidelines on the Conduct of Surveys of the Quality of Medicines. ${ }^{13}$

\section{SURVEY PERIOD}

Samples were collected from 45 public and private facilities across two regions and the city administration over the period of October 10-24, 2017.

\section{SELECTION OF SAMPLING AREAS}

The study collected samples from two regions and one city administration: Oromia, Afar and Addis Ababa respectively. The Oromia and Afar regions were selected as contrasting settings to provide information into geographical variations. Oromia is a large, better resourced region and has the highest population in the country. Afar is an emerging region that lacks infrastructure, endures high ambient temperatures and requires supply chains to accommodate large distances between supply hubs and healthcare facilities. As such, Afar represents a setting with the greatest barriers to maintaining oxytocin quality.

The selection of these regions ensured this study complemented the previous unpublished post-marketing activities conducted by the Ethiopian Food, Medicine and Healthcare Administration and Control Authority ((FMHACA), now the Ethiopia Food and Drug Authority, assessing oxytocin quality in Addis Ababa and five different regions (Tigray, Southern Nations, Nationalities and Peoples Region, Benishangul-Gumuz, Somali and Amhara). Within Oromia and Afar, collection sites in both urban and rural areas were targeted to understand within-region variations in quality that may arise due to disparities in cold chain reliability between urban and rural settings. Addis Ababa is the administrative centre of Ethiopia with greatest population density and serves as the entry point for all publicly procured oxytocin supplies and therefore constitutes the start of the public supply chain.

\section{SAMPLING DESIGN AND SELECTION OF SAMPLE COLLECTION SITES}

This section summarises the sampling methodology used based on a detailed operating procedure provided to the 
sampling teams (Appendix S1 in the Online Supplementary Document).

Drug products for public provision in Ethiopia are purchased and distributed by the Pharmaceutical Funding and Supply Agency (PFSA) within the FMoH. Oxytocin is not manufactured locally and so all product is internationally supplied, entering the country either via Addis Ababa airport or overland. This study aimed to sample material at the PFSA storage facilities at the airport, at the central storage facility and at regional hubs in Oromia and Afar, which, combined with samples from healthcare facilities supplied by these centres, would be used to assess quality of ampoules at all points along the public supply chain.

In each region, one regional PFSA distribution hub was selected and then public healthcare facilities supplied by the chosen regional hub were identified in accordance with the predetermined sampling schedule including hospitals and health centres. Private healthcare facilities were selected on the basis of proximity to selected public facilities to assist in the efficiency of collection. In addition, collection sites targeted included the PFSA storage facility at Addis Ababa airport (first port of entry) and the PFSA central store in Addis Ababa where public oxytocin supplies are stored immediately post-importation.

Samples were collected at all levels of the public healthcare system from tertiary referral hospitals to rural health centres. It was hypothesised that the consistency of cold chain supply and storage may decrease in lower tier facilities and rural settings where resources are fewer and power interruptions may be more frequent and protracted. In addition, private supply outlets and healthcare clinics were sampled to understand public versus private variations.

\section{SAMPLE COLLECTION AND TRANSPORTATION}

Sampling was conducted by collection team(s) comprising a minimum of two trained collectors using either an overt sampling or a mystery shopper strategy as appropriate. Overt sampling was used at all public facilities with prior approval from an appropriate facility representative. Importantly, the FMoH Maternal and Child Health Directorate provided a letter of authorisation that assisted in the approval at the facility level. Where appropriate (public facilities), samples collected for analysis were replaced by researchers with ampoules purchased prospectively PFSA. Samples collected from private facilities used a mystery shopper approach.

The sampling plan aimed to collect samples from 48 facilities. The target number of ampoules per sample was 25 ampoules to allow replicate assay testing, investigative work and microbiological (sterility and endotoxin) testing. If multiple batches were held at a facility, collectors would seek to collect a sample for every batch present. In cases where a limited number of ampoules were available, a minimum of nine ampoules were collected per sample.

Sample details such as facility information, number of ampoules, batch number, manufacturer information and storage conditions were recorded using a sample collection form (Appendix S2 in the Online Supplementary Document).
After collection, samples were retained in cool boxes until they were returned to Addis Ababa where they were stored under refrigerated conditions $\left(2-8^{\circ} \mathrm{C}\right)$. Once samples from all regions were available in Addis Ababa, samples were divided and shipped under ambient conditions to Monash Institute of Pharmaceutical Sciences (MIPS) and the Quality Control Laboratory at EFMHACA for analysis (total storage and shipping time $=24$ days). All samples received at these locations were stored at $2-8^{\circ} \mathrm{C}$ until the time of analysis. A temperature logger recorded the temperature of sample storage post-collection and during shipping to MIPS, and indicated no evidence of significant temperature excursions during this time.

\section{TESTING LABORATORIES}

Testing was conducted at the following three sites as described in Table 1:

- Medicine Quality Control Laboratory, EFMHACA, Addis Ababa, Ethiopia

- HMST Laboratory, MIPS, Melbourne, Australia

- Chemical Analysis Ltd, Melbourne, Australia

\section{QUALITY TESTS PERFORMED AND TEST METHODS AND SPECIFICATIONS USED}

Sample testing was conducted in accordance with Table 1. Where incomplete samples were collected ( $<25$ ampoules), assay testing was prioritised and microbiological testing conducted on a discretionary basis. Microbiological testing was conducted per batch ie, where a single batch was collected from multiple sites only one sample was used for microbiological testing.

\section{DEFINITION OF COMPLIANCE OF SAMPLES WITH STANDARDS}

All samples found to be out-of-specification were retested to verify results obtained. All data was subject to review and approval by quality control functions at all testing sites.

\section{RESULTS}

\section{OVERVIEW OF SAMPLES COLLECTED}

Complete samples of 25 ampoules were collected from 39 facilities while reduced numbers of ampoules were collected from a further six facilities. All samples comprised a minimum of 10 ampoules. All samples collected comprised $1 \mathrm{~mL}$ ampoules labelled to contain 10 IU oxytocin solution for injection.

\section{MANUFACTURERS AND BATCHES}

Samples collected were identified as being supplied from the following three manufacturers:

- RotexMedica GmbH (38 samples)

- Biologici Italia Laboratories S.r.l (4 samples)

- Novartis AG (3 samples)

Most samples comprised ampoules from a single batch of product from a given manufacturer, however samples 
Table 1. Summary of sample testing (full sample)

\begin{tabular}{|c|c|c|c|}
\hline Test & $\begin{array}{c}\text { Method/ } \\
\text { Specification }\end{array}$ & $\begin{array}{c}\text { No. of } \\
\text { Ampoules }\end{array}$ & Comments \\
\hline \multicolumn{4}{|l|}{ Testing at EFMHACA } \\
\hline Appearance & USP & 3 & Uses same ampoules as assay testing \\
\hline Labelling & USP & & \\
\hline Assay & USP & & Triplicate testing \\
\hline Assay (spare) & USP & 6 & $\begin{array}{l}\text { To allow repeat testing if first test found ampoules to be out } \\
\text { of specification }\end{array}$ \\
\hline \multicolumn{4}{|l|}{ Testing at MIPS } \\
\hline $\begin{array}{l}\text { Investigative Testing } \\
\text { (descriptive) }\end{array}$ & $\begin{array}{l}\text { In-house } \\
\text { methods }\end{array}$ & 3 & Testing to identify degradants/impurities \\
\hline \multicolumn{4}{|c|}{ Microbiological Testing } \\
\hline Validation (sterility) & USP $<71>$ & 8 & \multirow{2}{*}{$\begin{array}{l}\text { Microbiological testing subcontracted to Chemical Analysis } \\
\text { Ltd (Melbourne) }\end{array}$} \\
\hline $\begin{array}{l}\text { Sterility Testing } \\
\text { Endotoxin Testing }\end{array}$ & $\begin{array}{l}\text { USP }<71> \\
\text { USP }<85>\end{array}$ & 5 & \\
\hline Total & & 25 & \\
\hline
\end{tabular}

USP - United States Pharmacopeia, MIPS - Monash Institute of Pharmaceutical Sciences, EFMHACA - Ethiopian Food, Medicine and Healthcare Administration and Control Authority

Table 2. Number of facilities from which samples were collected in each region and area

\begin{tabular}{|c|c|c|c|c|}
\hline \multirow[t]{2}{*}{ Area } & \multirow[t]{2}{*}{ Facility type } & \multicolumn{3}{|c|}{ City/Region } \\
\hline & & Addis Ababa & Oromia & Afar \\
\hline \multirow{6}{*}{ Urban } & PFSA Hub & 1 & 1 & 1 \\
\hline & Public hospital & 3 & 3 & 1 \\
\hline & Public health centre & 3 & 3 & 3 \\
\hline & Private pharmacy & 3 & 3 & 1 \\
\hline & Private Hospital & 2 & - & - \\
\hline & Private Clinic & 1 & 3 & 1 \\
\hline \multirow{2}{*}{ Rural } & Public hospital & - & 3 & 1 \\
\hline & Public health centre & - & 3 & 5 \\
\hline
\end{tabular}

from five facilities comprised multiple batches from a single manufacturer.

\section{SITES OF SAMPLE COLLECTION}

Samples were collected from 45 facilities across the regions. The sites comprised hospitals, health centres, maternal and child health centres and pharmacies from across the public, private and NGO sectors in addition to regional PFSA storage hubs (Table 2). The following deviations from the proposed sample collection sites were necessary:

- Collection of samples from the PFSA storage facility at Addis Ababa airport was not possible as no oxytocin products were stored at this location at the time of the study.

- The protocol targeted three private healthcare facilities in Afar for sample collection. However, collection at this number of sites was not possible as the required number of oxytocin ampoules were available only at one out of six private healthcare facilities vis- ited. In order to maintain sample numbers, two additional public health centres in Afar were sampled.

\section{REGISTRATION STATUS OF SAMPLED PRODUCTS}

All samples were supplied from European-based, stringent regulatory authority approved manufacturers. However, at the time of sampling, the Novartis product was the only product registered by EFMHACA (according to the EFMHACA website). These samples comprised $7 \%$ of the samples collected during the study. The RotexMedica product was registered in December 2017 shortly after this survey was completed.

\section{STORAGE CONDITIONS OF SAMPLED PRODUCTS}

Where possible, the storage conditions at the time of collection were recorded. Thirty three samples were collected from functional refrigerators, while two samples were not stored in refrigerated conditions. The storage conditions of the remaining 10 samples could not be verified. 


\section{TESTING RESULTS}

Individual sample results are provided in Table S1 in the Online Supplementary Document. Samples from two sites did not meet the US pharmacopeial assay specifications (90-110\% of specified content) with the mean content of oxytocin being $78.8 \%$ and $78.3 \%$ of label claim in each sample. Both samples were collected from rural public health centres in Afar. Further analysis of these ampoules revealed the presence of known degradation products of oxytocin. The samples from these sites passed all other tests.

All other samples passed all tests.

\section{DISCUSSION}

\section{INTERPRETATION OF THE RESULTS}

The quality of the oxytocin samples collected during this study was generally high, in contrast to published studies conducted in other sub-Saharan countries. ${ }^{7}$ A contributing factor to these positive results is likely that all samples collected were supplied by European manufacturers approved by stringent regulatory authorities. In addition to the high manufacturing standards, all samples were labelled for refrigerated storage and the majority (at least 73\%) were stored in the cold chain at the time of collection, with only $4 \%$ of samples observed as being stored under ambient conditions. The Ethiopian Emergency Obstetric and Newborn Care (EmONC) Assessment Report (2016) details a review of 3780 healthcare facilities and observed a similar proportion that stored oxytocin in refrigerated conditions. ${ }^{14}$ This is largely in accordance with international recommendations, most specifically the recent joint WHO/United Nations Children Fund (UNICEF)/United Nations Population Fund (UNFPA) statement aimed at the procurement, supply and use of high quality oxytocin injection products. This statement recommends that all oxytocin products be procured from stringent regulatory or WHO approved suppliers and be stored in the cold chain throughout the distribution chain with labelling to that effect. ${ }^{3,15}$

Other studies conducted in resource limited settings have found that oxytocin ampoules have originated from manufacturers without stringent regulatory assessment and often with labelling that does not require refrigerated storage of the product. $6,8,16$ Qualitative studies among stakeholders have found that this labelling leads to confusion as to the relative stability of differently labelled products and undermines evidence that routine ambient storage risks product degradation, particularly in countries with hot climates (ie, the majority of low and middle income countries). ${ }^{17}$

The two samples from the current study that failed assay testing were collected at public rural facilities in Afar, a poorly resourced region with a very hot climate. Additional testing revealed the presence of oxytocin degradation products in these samples suggesting heat exposure. Given that samples collected from the PFSA regional hub in Afar passed testing, these samples were likely exposed to elevated temperatures during supply from the regional hub and/or storage at the facility. In one case the sample was stored in non-refrigerated conditions at the time of collection, which supports this hypothesis. The storage conditions of the second sample could not be verified.

Only $7 \%$ of samples collected (one of the three products found on the market) comprised a product that had been approved and registered by EFMHACA at the time of collection, including only one sample from public facilities. The mechanism by which the other products were procured and entered the country is probably through the stringent regulatory authorities approved products recognition scheme but the reasons why these products were not in the approved medicines list is unknown and warrants further investigation. It is possible that unregistered products can be procured legally under waiver arrangements to cover stock shortages, but this would need to be verified.

\section{RECOMMENDATIONS}

While the quality of oxytocin sampled in this study was high, the incidence of PPH in Ethiopia also remains high. Therefore, given the small geographical scope of the study, further investigations are warranted to verify these results on a national basis.

The data collected in Afar indicates that the public supply chain in this region may present a risk to oxytocin injection quality at the point of use, and a review of these systems should be prioritised. Similarly, a wider review should take a risk-based approach and focus initially on regions of least resource with high ambient temperatures and those with high maternal mortality due to $\mathrm{PPH}$.

Ninety-three percent (93\%) of samples (2/3 of the products) collected were unregistered at the time of collection. Further investigations are recommended to understand the provenance of these products and review procurement procedures in both public and private sectors.

\section{LIMITATIONS OF METHODOLOGY}

One limitation of this study is that the number of sample sites and the geographical spread of sample locations within each region was small. Therefore, these results should be considered indicative of those locations, but are not representative of the regional or national situation. The sample sites of this study should be used to guide the design of future studies to identify geographic gaps where further investigation is warranted to provide a more comprehensive picture of nation-wide oxytocin quality.

Overt sampling was conducted at public facilities and it was not always possible for collection teams to witness from where within the facility samples were being provided. This represents a second limitation as it was not always possible to confirm that the samples and their reported or observed storage conditions were representative of the stock available. For example a recent qualitative study found that in one facility a small batch of oxytocin ampoules were storage refrigerated, while the larger facility stock was stored at room temperature. ${ }^{17}$

It should also be noted that the data collected about oxytocin storage conditions in this study presents only a single time point of information. While these data do speak to the knowledge and practice of stakeholders towards refrig- 
erated storage of oxytocin, limited inferences can be made about the temperatures the products had been exposed to from the point of entry until the time of collection. Although it was beyond the scope of the current study, research to understand the time-course of temperatures associated with oxytocin supply and storage is warranted.

At five locations, insufficient ampoule numbers were collected to allow the conduct of microbiological testing. It was outside the scope of this study to understand why limited ampoule numbers were available at certain sampling sites, but further investigation may be warranted.

\section{CONCLUSIONS}

This study indicates that the quality of oxytocin at the locations sampled in Ethiopia is high, with oxytocin ampoules universally supplied from European-based, stringent regulatory authority approved manufacturers. All supplies were labelled for refrigerated storage with most being stored accordingly in facilities at $2-8^{\circ} \mathrm{C}$ in accordance with recent WHO/UNICEF/UNFPA recommendations. However, there are indications that deficiencies may exist in cold chain infrastructure and/or supply and storage procedures in rural resource-constrained areas with the potential to compromise oxytocin quality.

A review of procurement and registration procedures is warranted to understand the high proportion of product sampled during this study that was unregistered by EFMHACA.

Further post-marketing surveillance studies assessing additional collection sites and regions are required to understand the quality of oxytocin supplies in other regions of Ethiopia to better understand the national situation.

\section{ACKNOWLEDGEMENTS}

The authors gratefully acknowledge the support of the Ethiopian FMoH Maternal and Child Health Directorate and all participating facilities in the conduct of this study. The study design was approved by the Institutional Review Board of St Paul's Hospital Millennium Medical College (reference number PM23/76).

\section{FUNDING}

The study was funded through a grant to Monash University from the Reproductive Health Supplies Coalition Innovation Fund (ref. MAC.2042-01361263-GRT) with supplementary funding from an unrestricted grant to Monash University from the McCall MacBain Foundation.

\section{COMPETING INTERESTS}

The authors completed the Unified Competing Interest form at http://www.icmje.org/coi_disclosure.pdf (available on request from the corresponding author). MPM is the coinventor of a patent describing a novel, heat stable, powder formulation of oxytocin. PL, VLO, MPM, THN, AJLM and CG were supported by funding from the Reproductive Health Supplies Coalition and the McCall MacBain Foundation. AMT and BB received funding support from the Reproductive Health Supplies Coalition through Monash University. ZM and DB declare no competing interests.

\section{CORRESPONDENCE TO:}

Professor Michelle McIntosh, PhD

381 Royal Parade

Parkville VIC 3052

Australia

michelle.mcintosh@monash.edu 


\section{REFERENCES}

1. Ozawa S, Evans DR, Bessias S, et al. Prevalence and Estimated Economic Burden of Substandard and Falsified Medicines in Low- and Middle-Income Countries: A Systematic Review and Meta-analysis. JAMA Netw Open. 2018;1(4):e181662. doi:10.1001/ja manetworkopen.2018.1662

2. World Health Organization. WHO

Recommendations: Uterotonics for the Prevention of Postpartum Haemorrhage. World Health Organization; 2018.

3. WHO, UNICEF, UNFPA. Appropriate Storage and Management of Oxytocin - a Key Commodity for Maternal Health; 2019.

4. Schocken C. Business Case: Investing in Production of High-Quality Oxytocin for Low-Resource Settings. JHPIEGO; 2014.

5. Appiah B. US Pharmacopeia fighting counterfeit medicines in Africa. Can Med Assoc J. 2013;185(14):E666. doi:10.1503/cmaj.109-4571

6. Lambert P, Nguyen TH, McEvoy C, et al. Quality of oxytocin ampoules available in health care facilities in the Democratic Republic of Congo: an exploratory study in five provinces. $J$ Glob Health. 2018;8(2):020415. doi:10.7189/jogh.08.020415

7. Torloni M, Gomes Freitas C, Kartoglu U, Metin Gülmezoglu A, Widmer M. Quality of oxytocin available in low- and middle-income countries: a systematic review of the literature. BJOG: Int J Obstet Gy. 2016;123(13):2076-2086. doi:10.1111/1471-052 8.13998

8. Anyakora C, Oni Y, Ezedinachi U, et al. Quality medicines in maternal health: results of oxytocin, misoprostol, magnesium sulfate and calcium gluconate quality audits. BMC Pregnancy Childbirth. 2018;18(1):44. doi:10.1186/s12884-018-1671-y

9. Say L, Chou D, Gemmill A, et al. Global causes of maternal death: a WHO systematic analysis. Lancet Glob Health. 2014;2(6):e323-e333. doi:10.1016/s221 4-109x(14)70227-x
10. WHO, UNICEF, UNFPA. Trends in Maternal Mortality: 1990 to 2015; 2015.

11. EPHI. National Report on MDSR Data From 2006-2007 Ethiopian Financial Year. Ethiopian Public Health Institute; 2016.

12. Admasu KB. Health Sector Transformation Plan: 2015/16 - 2019/20 (2008-2012 EFY). Federal Government of the Democratic Republic of Ethiopia; 2015.

13. WHO. Guidelines on the Conduct of Surveys of the Quality of Medicines. World Health Organization; 2016.

14. Ethiopian Public Health Institute, Federal Ministry of Health, Columbia University. Ethiopian Emergency Obstetric and Newborn Care (EmONC) Assessment 2016. Ethiopian Public Health Institute, Federal Ministry of Health, Columbia University; 2017.

15. PATH, USAID, Coalition RHS. Buy Quality Oxytocin, Keep It Cold: An advocacy messaging framework for oxytocin, the first-line drug for the prevention and treatment of post-partum hemorrhage; 2018.

16. Karikari-Boateng E. Post-Market Quality Surveillance Project: Maternal Healthcare Products (Oxytocin and Ergometrine) on the Ghanaian Market. Ghana Food and Drugs Authority (FDA) Laboratory Services Department \& The Promoting the Quality of Medicines Program; 2013.

17. Oliver VL, Lambert PA, Than KK, et al. Knowledge, perception and practice towards oxytocin stability and quality: A qualitative study of stakeholders in three resource-limited countries. PLOS ONE. 2018;13(9):e0203810. doi:10.1371/journal.pone.0203 $\underline{810}$ 


\section{SUPPLEMENTARY MATERIALS}

\section{Online Supplementary Document}

Download: https://www.joghr.org/article/12112-oxytocin-injection-quality-in-ethiopia-a-post-marketing-surveillancestudy-in-public-and-private-facilities-across-three-regions/attachment/34009.pdf 